\title{
Zur Geschichte der Milchzuckergewinnung, besonders in der Schweiz
}

\author{
Von E. Bircher, Aarau
}

Wie Funk zutreffend ausführt, kommt dem italienischen Arzt und Gelehrten Fabrizio Bartoletti ${ }^{1}$ das Verdienst zu, das wirksame Agens in der Milch, das auch in der Molke das wichtigste ist, das Saccharum lactis, das er manna seri oder sal sai seri essentiale nennt, festzustellen.

Als Milchzucker, Saccharum lactis (Sale di sero dolcificato), wird er von dem Venezianer Lodovico Testi ${ }^{2}$ als Geheimmittel in den Arzneischatz eingeführt. Dieser Milchzucker wurde scheinbar hauptsächlich aus der Molke gewonnen und war beinahe eine Panazee in der Behandlung der Gicht.

1698 kam der Jenenser Arzt J.S. Seidel durch einen reisenden Italiener in den Besitz des Mittels, ohne es weiter anzuwenden. Erst als es von einem Arzte, Dr. Werloschnigg, bei der Gicht eines Zisterziensermönches mit gutem Erfolg angewandt worden sei, wurde es in Deutschland allgemein bekannt und mußte teuer bezahlt werden. Schon damals wurde aus solchen Präparaten - genau wie heute - möglichst viel Geld geschlagen (Näheres bei FunK ${ }^{3}$ ).

Der bernische Ökonom KöNIG schrieb 1705 in seiner Georgica helvetica curiae, daß die Molken, Schotten aus Käsewasser, eine gute Arznei seien, um aufwallendes Blut zu kühlen, für Fieber und Gliederkrankheiten, wenn man mit Tanae-Rinde, Zitronensaft oder Weinstein scheide. Als Zusatz wird Löffelkraut, Sauerampfer, Brunnenkresse, Biberklee verwendet. Reum oder Lupp wird nach KöNıG wie folgt hergestellt: Einem Kalbsmagen wird die innere (Käse-) Kruste abgekratzt oder die Körner von der Milch (Drüsengewebe) und sauber gewaschen. Die Kruste (Käse), ein Löffelein feinstes Mehles, ein Ei und etwas Saffran, ein Löffelein trinkwarme Milch durch-

${ }^{1}$ Bartoletti, geboren am 7. September 1576 in Bologna, gestorben am 3. April 1630 in Lendinara an der Pest.

2 Testi, geboren $1640 \mathrm{zu}$ Reggio, Arzt in Venedig, gestorben am 3. September 1707 in Venedig. Das Geheimmittel (!) ging an die Familie Testı über, die das Manuskript seinem Vetter D. Fulvio und seiner Base Gerhulda vermachte, der es durch den Arzt Antonio Vallisneri, Bologna, geboren am 3. Mai 1661 in Modena, gestorben am 18. Januar 1730, in Padua, weitergab.

${ }^{3}$ Erich Funk, Geschichtliches über Molke und Milchzucker, Die Milchwissenschaft, Heft 6, Nürnberg, Juni 1948. 
einander gemischt, in den sauber gewaschenen Kälbermagen eingefügt. Das ganze wird für einige Tage ins Kamin gehängt, bis es sich wie Brot schneiden läßt. Die Arbeiten von KöNig dürften Albreght von Haller nicht unbekannt gewesen sein. Die ersten Milchanalysen scheinen (nach FunK) zuerst von BERZELIUS 1814 und nachher von BERTHOLET 1870 veröffentlicht worden zu sein.

Der Milchzucker wurde 1780 von Carl Wilhelm Scheele genauer chemisch untersucht und bestimmt.

Die Milchsäure hat Bergmann 1799 als Säure angesehen, was aber erst von Mitscherlich und Liebig definitiv festgestellt wurde.

Über die systematische Zuckergewinnung im Entlebuch berichtet Merz in Faido ${ }^{4}$ in einer sehr aufschlußreichen Schrift. Er berichtet, daß 1787 ein Küher in Marbach ${ }^{5}$, der ein Käsetuch aufgehängt hatte, an Stelle der Schotte weiße Kristalle vorfand, sogenannten zuckerhaltigen Sand. Dieser wurde als Medikament nach BARToLETTI betrachtet, das im Laufe der Jahre bis 1887 zehn Millionen Franken in das kleine Voralpendörfchen brachte.

So wurden dann die Schotten (Molken) eingedampft die Gerinnsel mit Albulmanten; zuerst entstand Sirup, durch weiteres Eindampfen konnte man Zuckertafeln erhalten, für die ein höherer Preis bezahlt wurde. 1810-1820 brachte es die Konkurrenz zu einer Preistreiberei, der Zucker stand auf 25 Franken, fiel auf 10 Franken (1830), um wieder von neuem anzusteigen, 1856 auf 35 Franken, Ende der siebziger Jahre auf 135 Franken, 1880 gingen 460000 Franken in das Dorf. 1880 gab es fünfzehn Fabrikanten (Käser) für Zucker in Marbach. Nun befaßte sich die Industrie damit. Die Firma Gerber in Steffisburg nahm sich der Sache an, ebenso eine Fabrik in Merlach bei Murten. Neben Molkenbrot (2 1 Molke auf $500 \mathrm{~g}$ Weizen) wurde auch Molkenspiritus und Molkenessig hergestellt. Das Einsieden der Molke geschah in den Käsereien an einem bestimmten Ort, dem Feuergrübeli. Für $100 \mathrm{~kg}$ Zuckersand benötigte man $4 \mathbf{m}^{3}$ Holz. Man ließ das Eingedickte zweimal 24. Stunden an einem kühlen Orte stehen. Die Alpen-

${ }^{4}$ Merz (Faido), Das Entlebuch und seine Viehzucht. Dr. Portmann und Nationalrat STUDER in Escholzmatt machten mich auf diese Industrie aufmerksam.

${ }^{5}$ Marbach (Luzern), $880 \mathrm{~m}$ über Meer, an der Straße Langnau über den Schallenberg, $5 \mathrm{~km}$ südlich der Station Wiggen, Linie Bern-Luzern, am Fuße der Schrattenfluh, 1506 Einwohner, 1470 Luzern zugesprochen, 1808 verbrannt (Historisch-Geographisches-Lexikon), 1929). Noch bis zum Jahre 1890 lieferte die Zuckerfamilie KocH in Marbach nach Darmstadt, Hamburg und anderen deutschen Städten Milchzucker, zuletzt an die heute noch bestehende blühende Industriefirma Siegfried in Zofingen. 
milch eignete sich zur Herstellung besser als die Milch aus der Ebene; süße Molke geeigneter als saure. Die Qualität der Weide und des Futters war wichtig (Kräuter!).

Schottenzucker wurde in ähnlicher Weise im Entlebuch, Toggenburg, Emmental, Kiental, in Hasle-Frutigen, Freiburg, Arth am Zugersee hergestellt.

In Schangnau ${ }^{6}$ im Bernbiet wurde die Zuckerfabrikation ebenfalls betrieben. Dort verkauften die Lehenhüter, die keine Schweinehaltung trieben, die Schotte oder den Schottensand eingesotten an die sogenannten Schottenzuckersieder, die diesen Sand mit einer kleinen Beimischung von Alaun in kristallisierten Schottenzucker verwandelten, der während der Französischen Revolution in großen Handel kam und bis 1801 ein gutes Geschäft war, dann aber ganz aufhörte und wiederum mehr der Schweinezucht Platz machte.

Das Holzproblem war das Entscheidende. Zunächst wurde damit verschwenderisch umgegangen, auch für die Käsereien; mußte es herantransportiert werden, wurden die Kosten erheblich vermehrt.

Der Zuckersand wurde gewaschen und getrocknet, da man das Raffinieren noch nicht kannte. Das Eindampfen dauerte bis zu 16 und 22 Stunden. Aus 12001 Molken konnten $25 \mathrm{~kg}$ Zuckersand gewonnen werden. Der Rückstand der Schotten fand in der Schweinemast Verwendung.

Der Holzverbrauch, dessen Fällen, Rüsten und der Transport mit einem bis zwei Pferden oder Maultieren erhöhte die Kosten. Die Herstellung war mehr oder weniger eine Holzfrage. Da der Milchzucker vielerorts durch den Rohrzucker immer mehr verdrängt wurde, zeigte sich bald, daß die Schweinemast erheblich rentabler war, indem auf $60 \mathrm{Kühe} 20 \mathrm{Schweine}$ gehalten wurden. Die Napoleonische Kontinentalsperre war in der Lage, die Milchzuckerfabrikation zu fördern.

Die Raffinierung geschah in den Fabriken. Zwei Drittel Wasser, $30^{\circ} \mathrm{R}$, mit einem Drittel Zuckersand; die Mischung wurde ins Wallen gebracht. Zur Klärung brauchte man auf $10011 \mathrm{~kg}$ Alaun oder schwefelsaure Tonerde, Filterung mit Knochenkohle. Der Sirup mußte blättlen, der sogenannte «Sutt». So erhielt man aus $100 \mathrm{l}$ Molke 1,2 bis 1,5 kg Milchzucker. Die billigeren Kosten, die durch die Herstellung aus dem pflanzlichen Zuckerrohr ermöglicht wurden, erledigten die Fabrikation des tierischen Milchzuckers vollends. Milchzucker wurde noch in der Medizin,

${ }^{6}$ Josef Odermatt (Stans), Die Emmentaler Alpen, Schürch Söhne, Huttwil 1926. 
hauptsächlich in der Homöopathie und in der Säuglingspflege, verwendet (Dr. Eggeling). Hiezu berichtet Sekundarlehrer Dahinden:

«Mit der Alpwirtschaft im Zusammenhang steht auch die Bereitung des sogenannten Zuckersandes oder Schottenzuckers, welcher hauptsächlich auf den Alpen bereitet wurde. Dadurch wurde der Alp- und Milchwirtschaft eine bedeutende Erwerbsquelle erschlossen. Schon Pfarrer STALDER erwähnt diese, indem er schreibt : ,Öfters, wenn Waldung auf einem Alpberg im Überfluß dasteht und das Holz nicht mit Vorteil kann verkauft werden, kocht man hie und da Schottenzucker, der auch ins Ausland verkauft wird.' Die Heimat der Schottenzuckerfabrikation und der Herstellung des kristallisierten Milchzuckers ist Marbach, von wo sie auf unsere Alpen den Weg fand. »

F. MERz schreibt darüber: «In Marbach befanden sich acht Milchzuckerfabrikanten. »

Auch J. PEter, Handelsmann in Schüpf heim, beschäftigte sich mit dem Handel von Zuckersand und kristallisiertem Milchzucker. Die südlich gelegenen Alpen lieferten den Zuckersand auf dem kürzeren Wege über Kemmeriboden nach Marbach, ebenso Schwandalp in Flühli. Dieses Zuckersieden geschah in eigens dafür erstellten primitiven Hütten, den sogenannten Zuckerhütten. Für einen Sud braucht es viel Zeit, 16-20 Stunden, je nach Quantum, und sehr viel Brennmaterial, man rechnet auf $100 \mathrm{~kg}$ Zuckersand ungefähr zwei Klafter Holz, welches aber um jene Zeit sehr billig war. Diese Industrie konnte naturgemäß nur bei guten Preisen rentieren. Diese schwankten zwischen 30, 40,60,70 Franken, je nach den Zeitverhältnissen und der Konkurrenz, und erreichten in den achtziger Jahren den höchsten Preis von 130 Franken. Ältere Älpler behaupten, sie hätten in diesen Zeiten des Höchstpreises von Zuckersand aus demselben so viel Gewinn gezogen wie aus dem Käse. Dann ging's finanziell aber infolge Konkurrenz modern eingerichteter Fabriken, welche eine bessere Ausbeute ermöglichten und das Produkt billiger liefern konnten, zurück, bis dieser Erwerbszweig in den neunziger Jahren vollständig einging. So wurde u.a. auf den Alpen Salzbühl und Bäregg von 1876 bis 1892 Schottenzucker gekocht. Die Zuckerhütten dienten später noch als Holzschuppen und sind jetzt gänzlich verschwunden. Außer den Älplern befaßten sich auch hie und da Private mit der Zuckersiederei, so in Flühli in den achtziger Jahren Josef Bucher in der Pfaffrüti mit der Herstellung von kristallisiertem Milchzucker (Zapfen und Blättchen). Die Unternehmung fiel aber in ungünstige Zeit. Es trat infolge Überproduktion ein Preissturz ein, so daß das Geschäft nach kurzer Zeit einging. - Nach der bestimmten Meinung 
von alt Nationalrat Balmer (Schüpfheim) hat die Publikation von Merz der Milchzuckerfabrikation in unserem Lande sehr geschadet. Durch die Bekanntmachung des Herstellungsverfahrens, das in unserem Lande, besonders in Marbach, fast als Geheimnis gehütet wurde, wurde dieses der breiten Öffentlichkeit zugänglich. Es ging nicht lang, so entstanden nicht nur in der Schweiz, sondern auch im Ausland, besonders in Deutschland, Zuckerraffinerien » (aus: Blätter für Heimatkunde aus dem Entlebuch 9, 553, 1936; von † Sekundarlehrer Jos. Dahinden, durch freundliche Vermittlung von Dr. PortmanN).

Der Vollständigkeit halber sei darauf hingewiesen, daß in Amerika, Schlesien, Ungarn und Holstein die Milchzuckerfabrikation Eingang gefunden hatte.

Jak.-Gerh. Reinhard Andreae aus Hannover, Apotheker, berichtet in seiner Schweizer Reise davon 1765 und ist bereit, solchen Zucker kommen zu lassen, denn dessen Herstellung hatte schon 1780 eine größere Ausdehnung zu medizinischen Zwecken gefunden. Medicus fand sie 1794 noch auf seiner Schweizer Reise speziell im Entlebuch und im Emmental. Zentrum war die Alp Marbach, auf welcher der Zuckersand geläutert wurde ${ }^{7}$. Auch Medicus mußte bestätigen, daß die Milchzuckerfabrikation von der Beschaffung des Holzes abhängig war, wenn er sagt: «Seine Verfertigung ist besonders nur auf solchen Alpen vorteilhaft, auf denen viel Holz wächst. »

Über diesen Handel mit dem Milchzucker berichtete Frank Roux 1772 im «Journal de Medicine ». 1772 erschien die erste Monographie LiechtenSTEINERS über den Milchzucker, dem auch die Molken bekannt waren und der dem Milchzucker im Serum die Heilkraft zuerkannte und auf die günstige therapeutische Wirkung, speziell auch bei der Tuberkulose, hinwies ${ }^{8}$.

Es sind jetzt hundertfünfzig Jahre her, seit in Schlesien die industrielle Rübenzuckerfabrikation in Betrieb genommen wurde, die zunächst mit großen Schwierigkeiten zu kämpfen hatte, während der Rohrzucker schon seit Alexander dem Großen (377 v. Chr.) bekannt war und seit dem Jahr 100 v. Chr. in Ägypten industriell ausgebeutet wurde ${ }^{9}$.

${ }^{7}$ Hofrat Ludwig Wallraht (Medicus aus Mannheim), Bemerkungen über die Alpenwirtschaft, Graeff, Leipzig 1795.

${ }^{8}$ Vgl. auch E. Bircher, Die Molkenkur, ihre Geschichte und geographische Verbreitung, Schweiz. Med. Wschr. 1953, 937; Fortsetzung 1954.

${ }^{9}$ Urs DefFner, Ein süßer Jubilar (150 Jahre Rübenzucker), Leben und Umwelt 8, 183 (1952). 